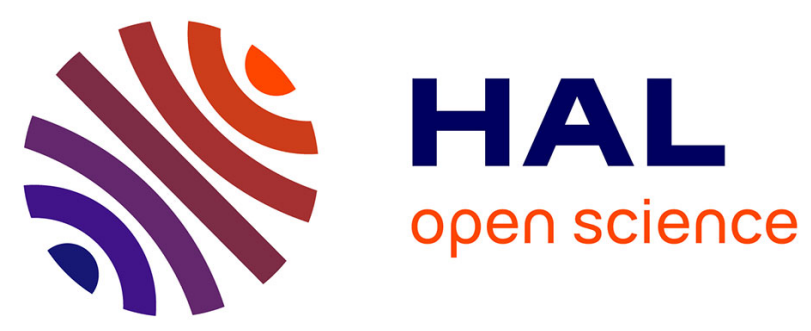

\title{
Factors associated with outcome and duration of therapy in outpatient parenteral antibiotic therapy (OPAT) patients with skin and soft-tissue infections
}

\author{
R.A. Seaton, E. Sharp, V. Bezlyak, C.J. Weir
}

\section{- To cite this version:}

R.A. Seaton, E. Sharp, V. Bezlyak, C.J. Weir. Factors associated with outcome and duration of therapy in outpatient parenteral antibiotic therapy (OPAT) patients with skin and soft-tissue infections. International Journal of Antimicrobial Agents, 2011, 38 (3), pp.243. 10.1016/j.ijantimicag.2011.05.008 . hal-00722868

\section{HAL Id: hal-00722868 \\ https://hal.science/hal-00722868}

Submitted on 6 Aug 2012

HAL is a multi-disciplinary open access archive for the deposit and dissemination of scientific research documents, whether they are published or not. The documents may come from teaching and research institutions in France or abroad, or from public or private research centers.
L'archive ouverte pluridisciplinaire HAL, est destinée au dépôt et à la diffusion de documents scientifiques de niveau recherche, publiés ou non, émanant des établissements d'enseignement et de recherche français ou étrangers, des laboratoires publics ou privés. 


\section{Accepted Manuscript}

Title: Factors associated with outcome and duration of therapy in outpatient parenteral antibiotic therapy (OPAT) patients with skin and soft-tissue infections

Authors: R.A. Seaton, E. Sharp, V. Bezlyak, C.J. Weir

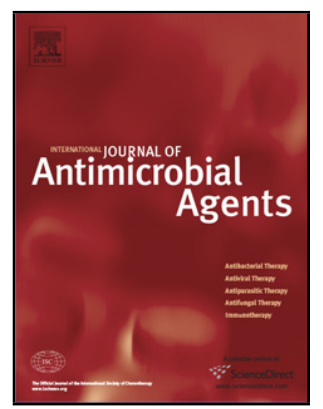

PII:

S0924-8579(11)00239-1

DOI: doi:10.1016/j.ijantimicag.2011.05.008

Reference: ANTAGE 3633

To appear in: International Journal of Antimicrobial Agents

Received date: $\quad$ 15-3-2011

Accepted date: $\quad$ 10-5-2011

Please cite this article as: Seaton RA, Sharp E, Bezlyak V, Weir CJ, Factors associated with outcome and duration of therapy in outpatient parenteral antibiotic therapy (OPAT) patients with skin and soft-tissue infections, International Journal of Antimicrobial Agents (2010), doi:10.1016/j.ijantimicag.2011.05.008

This is a PDF file of an unedited manuscript that has been accepted for publication. As a service to our customers we are providing this early version of the manuscript. The manuscript will undergo copyediting, typesetting, and review of the resulting proof before it is published in its final form. Please note that during the production process errors may be discovered which could affect the content, and all legal disclaimers that apply to the journal pertain. 
Factors associated with outcome and duration of therapy in outpatient parenteral antibiotic therapy (OPAT) patients with skin and soft-tissue infections is

R.A. Seaton ${ }^{a, k}$, E. Sharp ${ }^{a}$, V. Bezlyak ${ }^{b}$, C.J. Weir ${ }^{b, 1}$

${ }^{a}$ Brownlee Centre, Infectious Diseases Unit, Gartnavel General Hospital, NHS

Greater Glasgow and Clyde, 1053 Great Western Road, Glasgow G12 OYN, UK

${ }^{\mathrm{b}}$ Robertson Centre for Biostatistics, University of Glasgow, Glasgow, UK

ARTICLE INFO

Article history:

Received 15 March 2011

Accepted 10 May 2011

Keywords:

SSTIS

Cellulitis

Home therapy

Intravenous therapy

Ceftriaxone

Teicoplanin

Patient group direction 
* Corresponding author. Tel.: +44 141211 0292; fax: +44 1412111096.

E-mail address: andrew.seaton@ggc.scot.nhs.uk (R.A. Seaton).

${ }^{1}$ Present address: MRC Hub for Trials Methodology Research, Centre for Population Health Sciences, University of Edinburgh, Edinburgh, UK.

is This study was presented in part at the 20th European Congress of Clinical

Microbiology and Infectious Diseases (ECCMID), 10-13 April 2010, Vienna, Austria (abstract 1711). 


\section{ABSTRACT}

This study was designed to identify factors associated with adverse outcomes and increased duration of parenteral therapy in patients with skin and soft-tissue infections (SSTIs) managed with outpatient parenteral antibiotic therapy (OPAT). A retrospective cohort study interrogating variables recorded prospectively in an electronic OPAT patient database was performed. 'OPAT failure' was defined as hospitalisation following initiation of OPAT, or adverse event or progression of infection necessitating a change in antibiotic therapy. Variables associated with failure or increased duration of therapy were identified via univariate and multiple logistic regression analyses. In total, 963 first patient episodes of OPAT-treated SSTIs were observed; $84 \%$ were treated with daily ceftriaxone and $15 \%$ with teicoplanin (three daily loading doses then three times per week). Progression of infection was observed in $2.8 \%$ of cases, inpatient management was required in $6 \%$ and significant adverse events occurred in $7.1 \%$. Overall OPAT success was $87.1 \%$. Female sex, diabetes and treatment with teicoplanin were independently associated with OPAT failure. A significant reduction in duration of OPAT therapy was observed over time. A longer duration of intravenous therapy was associated with meticillinresistant Staphylococcus aureus (MRSA), older age, vascular disease, a diagnosis of bursitis, and treatment with teicoplanin. Non-inpatient referrals, management via a nurse-led patient group direction, and treatment with ceftriaxone were associated with reduced duration of OPAT. For selected patients with SSTIs, OPAT was generally safe and effective, but specific patient groups were identified with more complex management pathways and poorer outcomes. 


\section{Introduction}

In the UK, skin and soft-tissue infections (SSTIs) accounted for ca. $1.2 \%$ of acute hospital admissions in 2009/2010, with a rate of 174 per 100000 population [1]. Approximately one-half will require initial intravenous (i.v.) antibiotic therapy, amounting to $16 \%$ of all i.v. antibiotic-treated patients in hospital [2]. The duration of i.v. therapy varies markedly between centres, with a mean of 2.5-10 days [3-6] and an average hospitalisation of $4-13$ days [3-7]. Outpatient parenteral antibiotic therapy (OPAT) is increasingly used to simplify patient management and to shorten or avoid admission for patients with SSTIs [6,7]. As OPAT utilisation increases in mainstream healthcare in Europe, it is important to understand and anticipate factors that may be associated with poorer outcome or prolonged therapy in order to design and configure the service appropriately. Such factors have previously been considered in those with SSTIs managed as inpatients [3-5], however these may not be applicable to patients selected for management in an outpatient setting.

The Glasgow OPAT service, established in 2001, provides ambulatory i.v. or intramuscular therapy, administered by a team of specialist nurses, for patients with a variety of infections, referred either direct from the community (general practice or emergency departments) or following hospital admission. SSTIs comprise approximately one-third of OPAT patient referrals. Since July 2003, SSTI patients have been assessed via a nurse-led clinical protocol and selected patients have been managed with ceftriaxone via a standard patient group direction (PGD) [7]. Similarly, a PGD has also been developed and introduced for teicoplanin use since 2005. 
The purpose of the present observational study was, in the context of SSTIs, to retrospectively identify factors associated with OPAT failure and with longer i.v. treatment courses with a view to optimising future OPAT management strategies.

\section{Methods}

Patients with SSTIs are identified and referred either from community-based general practitioners, emergency department physicians or by hospital-based physicians. Initial OPAT assessment is by an experienced nurse specialist. Inclusion criteria for OPAT-managed SSTIs have been described previously [7]. Patients with rapidly progressive infection or sepsis criteria or other criteria for hospitalisation are excluded from initial OPAT therapy but may be referred after assessment and stabilisation in hospital. Otherwise, patients are treated with ceftriaxone $1 \mathrm{~g}$ daily as standard or with $2 \mathrm{~g}$ daily if weight $>70 \mathrm{~kg}$ or if extensive skin involvement. If patients have a $\beta$-lactam allergy or suspected meticillin-resistant Staphylococcus aureus (MRSA) infection, teicoplanin 10-15 mg/kg (daily loading for 3 days then thrice weekly) is used [8]. Patients are classified as having an MRSA infection if MRSA is isolated from a clinical isolate (e.g. pus or wound discharge) or patients were colonised with MRSA at the time of the SSTI. Patients are referred to infectious diseases medical staff at any time following initial referral if there is clinical deterioration/progression of the SSTI, development of a related or unrelated medical event, or any potential adverse event. Timing of i.v. to oral antibiotic switch is determined by specialist nursing staff as part of a locally agreed PGD [7]. The i.v. to 
oral switch can occur at any time following referral, including at the time of first review if criteria for i.v. therapy are not met.

Patients treated with ceftriaxone are switched by the specialist nurse to 7 days of either oral clindamycin 450-600 mg every $8 \mathrm{~h}$ or, if aged $>65$ years, flucloxacillin $0.5-$ $1 \mathrm{~g}$ every $6 \mathrm{~h}$ when evidence of improvement of clinical signs of SSTI [7]. Similarly, patients treated with teicoplanin switch to clindamycin (if penicillin allergy) or complete therapy with teicoplanin if MRSA infection (without oral switch) unless a clindamycin-sensitive MRSA has been isolated. Patients are advised to contact the OPAT service in the month following completion of therapy if there were concerns, recurrence or complications. Otherwise, routine clinic review is only arranged for patients with severe or recurrent infection. A patient satisfaction questionnaire is sent to all OPAT patients following completion of therapy and patients are encouraged to feedback on their OPAT experience. Outcome of the OPAT course is recorded following completion of therapy and is assigned by the specialist nurse in consultation with the infectious diseases consultant (R.A.S.).

Success of OPAT antibiotic treatment is recorded when there is clinical improvement allowing i.v. to oral switch and without recourse to further i.v. therapy or re-admission.

Failure of OPAT antibiotic treatment is recorded if the infection fails to improve or deteriorates despite OPAT. For the purposes of clinical audit and quality of care monitoring, an electronic database, wherein clinical information including outcome and adverse events is recorded, has been prospectively maintained. 
Patients with SSTI managed via OPAT between 2001 and 2008 were identified from the database. Case notes and patient management pathways were reviewed for the patient's first episode of OPAT only. Subsequent episodes of OPAT for individual patients were not included. Demographic data, details of prior hospitalisation, nature of infection, co-morbidities, details of therapy and outcome were recorded. For the purposes of this study, a more formalised definition of 'OPAT failure' was defined to incorporate aspects of both the response to antibiotic therapy and the overall clinical service. Thus, OPAT failure was strictly defined as hospitalisation following initiation of OPAT or adverse event or failure to improve or progression of infection necessitating a change in antibiotic therapy during OPAT and including relapse in the month following completion of OPAT.

Ethical approval for this study was not deemed necessary as the data were routinely collected and analysed for service development, clinical governance and quality assurance purposes.

\subsection{Statistical analysis}

Categorical variables are presented as number (\%) and continuous variables are presented as median with lower and upper quartiles. For the analysis of outcome, potential variables were first investigated using univariate analysis and then multiple logistic regression to model OPAT failure versus success. Results are presented as odds ratios, 95\% confidence intervals (Cls) and $P$-values. Variables associated with duration of OPAT were investigated using univariate and then multiple linear regression. Independent variables were log-transformed and the estimates and 95\% 
Cls were anti-log transformed. Estimates therefore have an interpretation in terms of percentage change in number of days in OPAT, e.g. an estimate of 1.10 means that a variable is, on average, associated with a $10 \%$ increase in the number of days of treatment. The purpose of multiple regression modelling was to identify the subset of variables that might be independently associated with each of outcome and OPAT duration. Teicoplanin and ceftriaxone were specifically investigated as these were the most commonly prescribed antibiotics.

\section{Results}

A total of 963 patients with a first episode of OPAT-treated SSTI were identified (Table 1). The majority were treated in OPAT initially with ceftriaxone $(84 \%)$ or teicoplanin (15\%). Eight patients received another antibiotic, including six (0.6\%) who received daptomycin. Of the ceftriaxone-treated patients, 650 (81.0\%) received $1 \mathrm{~g}$ and $152(19.0 \%)$ received $2 \mathrm{~g}$, with dosage data unavailable for 9 individuals.

Overall, 547 patients (56.8\%) were treated via the PGD [525 (64.7\%) of ceftriaxonetreated patients and $22(15.3 \%)$ of teicoplanin-treated patients]. There was a significant trend towards shorter duration of OPAT therapy over time (Fig. 1) (estimate per year $0.904,95 \% \mathrm{Cl} 0.886-0.922 ; P<0.0001$ ). This effect was observed in ceftriaxone-treated patients (estimate $0.907,95 \% \mathrm{Cl} 0.890-0.924 ; P<$ 0.0001) but not in the smaller group who received teicoplanin. Progression of infection was observed in 27 patients (2.8\%) (Table 2), with $8(29.6 \%)$ successfully managed via switch of antibiotic in the OPAT setting and the remainder requiring inpatient management. Admission or re-admission during OPAT occurred in $6 \%$ overall, the commonest reason being treatment failure with progression of infection or 
lack of response (Table 2). Significant adverse events were observed in 68 patients $(7.1 \%)$ and were more common in patients who received teicoplanin (Table 2). Drugrelated rash necessitating a change in antibiotic therapy was the most common adverse event (occurring in $9 \%$ of those receiving teicoplanin). Severe allergy or anaphylaxis was observed in three patients, all of whom received ceftriaxone. Gastrointestinal side effects, particularly diarrhoea, were the most frequent adverse events in ceftriaxone-treated patients (occurring in 1.8\%).

All patients with antibiotic-related diarrhoea submitted stool specimens for Clostridium difficile toxin detection, and infection was confirmed in one patient. In the remainder, diarrhoea resolved on discontinuation of antibiotic therapy. Overall, the OPAT success rate (no progression of infection, no re-admission and no significant adverse events) was $87.1 \%$ (839 patients).

In the univariate analysis, many factors were associated with OPAT failure, but only female sex, diabetes and treatment with teicoplanin were independently associated (Table 3). Several variables were noted to have univariate associations with duration of OPAT therapy (Table 4). In the multiple regression model, variables associated with prolongation of OPAT were MRSA infection, older age, vascular disease, diagnosis of bursitis, and treatment with teicoplanin (Table 4). Direct referral from the community or emergency department (rather than from an inpatient unit) and management via the nurse-led PGD were associated with reduced duration of OPAT (Table 4). No differences in duration of therapy or outcome were noted between patients treated with $1 \mathrm{~g}$ or $2 \mathrm{~g}$ of ceftriaxone (data not shown). 


\section{Discussion}

The aim of OPAT is to deliver high-quality ambulatory care that is at least equivalent to inpatient care for selected patients with serious infections. As such, it is essential to ensure that parenteral therapy is of the optimum duration (not unnecessarily prolonged or shortened) and is not associated with untoward adverse effects or poor outcome. Prospective maintenance of an electronic database and retrospective assessment of outcome in the Glasgow OPAT cohort has enabled us to describe a SSTI patient cohort and to evaluate the association between clinical and treatment variables and outcome. Potential limitations of the study include the retrospective assignation of 'OPAT failure', failure to record all possible clinical variables that may have impacted on clinical outcome (e.g. obesity) and the lack of formal longer-term follow-up of patients after completion of OPAT. Although some adverse events or readmissions may have occurred following the OPAT treatment course and potentially not been recorded on the database, various measures were in place to minimise this risk.

First, patients were advised to contact the OPAT service directly if there were any problems (including infection recurrence) in the month after the course of OPAT. Second, patients were sent a service evaluation questionnaire following the treatment course, where they were encouraged to feedback any problems.

Despite these limitations, to our knowledge this is the largest published cohort of OPAT-treated patients with SSTIs from a single centre. In nearly 1000 patients with SSTI managed via OPAT, the majority were middle aged, referred with 
cellulitis/erysipelas direct from the community and were managed via a nurse-led management protocol. 'OPAT failure' was strictly defined as one or more of progression of infection, switch to an alternative parenteral antibiotic, re-admission or significant adverse event. Diabetes, female sex and treatment with teicoplanin were all independently associated with increased risk of OPAT failure. In this pre-selected group of patients where those with the most complex co-morbidities were screened out and hospitalised, significant factors associated with prolonged OPAT included older age, vascular disease and more complex infections such as wound infection and bursitis.

Diabetes is a common risk factor for cellulitis [3-5,9] and poorer OPAT outcome may reflect a number of factors including poor vascularity, obesity, impairment of renal function, other related co-morbidities as well as complications of concomitant medications and potentially more advanced or complex infection. Diabetes has not been observed to adversely affect outcome in hospitalised patients with cellulitis [35] but a non-significant tendency for worse outcome was noted in one study comparing teicoplanin and cefazolin [9]. There is not an immediately clear biological reason why female sex should be an independent risk factor for OPAT failure and it is possible that this risk factor may have been confounded by other unidentified or unrecorded factors such as patterns of referral, severity of infection, unrecorded comorbidity or obesity. Notably, male sex was previously identified as a risk factor for poor outcome in a study of inpatients with cellulitis [5]. Other risk factors associated with mortality in hospitalised patients noted in that study included multiple comorbidities, renal impairment, shock, congestive cardiac failure, morbid obesity and pseudomonal infection [5], whilst older age, immobility or bed-bound status were 
identified by others [3]. Many of these factors (except age and obesity) would be contraindications for home therapy [7]. We identified only one published OPAT study (of 98 episodes of cellulitis with a mean duration of therapy of 6.3 days) that considered factors associated with prolongation of i.v. therapy [10]. That study lacked statistical power and categorised duration of treatment into two groups of $<6$ days or $\geq 6$ days [10]. We could not identify any studies that had investigated reasons for failure of OPAT as defined here.

Although this retrospective study was not designed to compare outcomes between ceftriaxone- and teicoplanin-treated patients, differences were noted. OPAT failure was observed in $25.7 \%$ of teicoplanin-treated patients compared with $10.5 \%$ of patients treated with ceftriaxone. OPAT failure was due to a combination of adverse events, re-admission and to a lesser extent progression of infection (Table 2) and was independent of MRSA infection, which was observed in only 47 patients (ca. $33 \%$ of teicoplanin-treated patients). Both teicoplanin treatment and MRSA infection were independently associated with prolongation of parenteral therapy. Greater patient complexity and other unrecorded clinical characteristics may have contributed to OPAT failure and prolonged therapy with teicoplanin. We previously noted the thrice-weekly teicoplanin dosing regimen was not associated with an increase in serious adverse events in a larger population of patients treated for a range of deepseated infections and SSTIs, many of whom had higher dosage of teicoplanin than in this cohort [8]. However, in the present study the single most important factor in teicoplanin-treated patients who failed on OPAT was rash, which occurred in $9 \%$ of patients. Although teicoplanin was not associated with infection treatment failure per se, duration of parenteral therapy was significantly longer despite daily review for the 
first three loading doses. There may have been opportunities for earlier oral switch in non-MRSA-infected patients (to clindamycin), which were missed because of the extended dosing interval after the loading doses. Intravenous to oral switch opportunities in MRSA patients similarly may have been missed but also because of the restricted availability of linezolid in our institution owing to higher drug acquisition costs. Slower clinical response to glycopeptides compared with ceftriaxone cannot be ruled out in this observational study. In previous SSTI studies comparing teicoplanin with cefazolin, success rates and duration of therapy were similar $[9,11]$. No difference in the rate of adverse events between the treatment groups was observed in one large study, with $8.9 \%$ of teicoplanin-treated patients withdrawing due to adverse events [9], but in a smaller study rates of adverse events were nonsignificantly higher in teicoplanin-treated patients (21\% vs. $3 \%)$ [11].

Duration of therapy (median 3 days) in the present study compared very favourably with other published experience. In a cohort of 124 patients treated with OPAT via an emergency department in Sydney, Australia, the mean duration of i.v. antibiotic therapy (with cefazolin) was 6.5 days and the re-admission rate was 15\% [12]. In 125 patients treated with OPAT (using either ceftriaxone or teicoplanin) in Dundee, UK, the mean duration of therapy was 5.3 days and the re-admission rate was reported at $2.4 \%$ [6]. In a five-centre retrospective review of 416 emergency departmentmanaged patients with cellulitis, ca. $80 \%$ received daily OPAT (mainly with cefazolin) for an average of 4 days. In that study, $11 \%$ required a change of antibiotic therapy and $7 \%$ required admission [13]. Once-daily cefazolin with oral probenecid or daily ceftriaxone administered in the outpatient setting were found to be equivalent in a 
randomised controlled trial in Australia (with $86 \%$ and $96 \%$ cure, respectively) and were associated with a mean of $6-7$ days of OPAT [14].

A decrease in the duration of i.v. therapy over time was observed. This corresponded with the development of the service, growing OPAT clinical experience and in particular the introduction of the nurse-led protocol and PGD in 2003. Overall, PGDs were utilised in $56.8 \%$ of patients and particularly in patients treated with ceftriaxone, $64.7 \%$ of whom were managed via the PGD. Daily treatment with ceftriaxone (with daily clinical review) was independently associated with a reduction in the duration of i.v. therapy over time. Management via the nurse-led PGD and treatment of patients presenting from the community without prior hospital admission were also associated independently with a reduction in the duration of i.v. therapy. These three factors probably combine to reflect increasing expertise by nurse specialists in identifying lower-risk patients suitable for earlier switch and likely explain the observed shorter duration compared with previously published reports $[6,10,12,13]$.

Failure of OPAT as defined by re-admission or antibiotic switch (owing to failure to improve or adverse event) was independently associated with female sex, diabetes and treatment with teicoplanin. Shorter duration in OPAT over time may reflect less severe infections referred from the community and managed via the nurse-led protocol, or increasing expertise in specialist nurses in identifying patients for earlier i.v. to oral switch.

Despite the convenience of thrice-weekly dosing of teicoplanin (following 3 days of loading) and experience to date in deep-seated infections [8], it is possible that this 
regimen may be less effective/efficient or less well tolerated for patients with SSTIs managed in the OPAT setting. Daily teicoplanin dosing (and review) may potentially optimise earlier i.v. to oral switch (to either clindamycin or linezolid). Alternatively, other daily administered parenteral agents such as daptomycin could be considered.

\section{Acknowledgment}

The authors are grateful to Lindsay Semple for assistance in data collection.

\section{Funding}

This study was funded by an unrestricted grant from Novartis, UK.

\section{Competing interests}

RAS has served as an advisor to and spoken at symposia sponsored by Novartis and Pfizer. CJW has acted as a consultant for Pfizer. ES and VB declare no competing interests.

\section{Ethical approval}

Not required. 


\section{References}

[1] NHS National Services Scotland. Incidence of hospital episodes by main diagnosis and financial year; and rates per 100,000 http://www.isdscotland.org/Health-Topics/Hospital-Care/Diagnoses/ [accessed 8 June 2011].

[2] Seaton RA, Nathwani D, Burton P, McLaughlin C, MacKenzie AR, Dundas S, et al. Point prevalence survey of antibiotic use in Scottish hospitals utilising the Glasgow Antimicrobial Audit Tool (GAAT). Int J Antimicrob Agents 2007;29:6939.

[3] Tan R, Newberry DJ, Arts GJ, Onwuamaegbu ME. The design, characteristics and predictors of mortality in the North of England Cellulitis Treatment Assessment (NECTA). Int J Clin Practice 2007;61:1889-93.

[4] Morpeth SC, Chambers ST, Gallagher K, Framptom C, Pithie AD. Lower limb cellulitis: features associated with length of hospital stay. J Infect 2006;52:23-9.

[5] Carratalà J, Rosón B, Fernández-Sabé N, Shaw E, del Rio O, Rivera A, et al. Factors associated with complications and mortality in adult patients hospitalized for infectious cellulitis. Eur J Clin Microbiol Infect Dis 2003;22:151-7.

[6] Nathwani D. The management of skin and soft tissue infections: outpatient parenteral antibiotic therapy in the United Kingdom. Chemotherapy $2001 ; 47($ Suppl 1):17-23.

[7] Seaton RA, Bell, E, Gourlay Y Semple L. Nurse-led management of uncomplicated cellulitis in the community: evaluation of a protocol incorporating intravenous ceftriaxone. J Antimicrob Chemother 2005;55:764-7. 
[8] Lamont E, Seaton RA, Macpherson M, Semple L, Bell E, Thomson AH. Development of teicoplanin dosage guidelines for patients treated within an outpatient parenteral antibiotic therapy (OPAT) programme. J Antimicrob Chemother 2009;64:181-7.

[9] Stevens DL. Teicoplanin for skin and soft tissue infections: an open study and a randomized, comparative trial versus cefazolin. J Infect Chemother 1999;5:40-5.

[10] Lillie PJ, Andrews D, Eaves K, Darton TC, Chapman ALN. Baseline factors predicting the duration of intravenous antibiotic therapy for cellulitis in an outpatient setting. Eur J Clin Microbiol Infect Dis 2010;29:347-9.

[11] Chirurgi VA, Edelstein H, Oster SE, Karp R, Cassano KB, Aiken S, et al. Randomized comparison trial of teicoplanin i.v., teicoplanin i.m., and cefazolin therapy for skin and soft tissue infections caused by Gram-positive bacteria. South Med J 1994;87:875-80.

[12] Donald M, Marlow N, Swinburn E, Wu M. Emergency department management of home intravenous antibiotic therapy for cellulitis. Emerg Med $\mathrm{J}$ 2005;22:715-7.

[13] Dong SL, Kelly KD, Oland RC, Holroyd BR, Rowe B. ED management of cellulitis: a review of five urban centres. Am J Emerg Med 2001;19:535-40.

[14] Grayson ML, McDonald M, Gibson K, Athan E, Munckhof WJ, Paull P, et al Once-daily intravenous cefazolin plus oral probenecid is equivalent to once-daily intravenous ceftriaxone plus oral placebo for the treatment of moderate-to-severe cellulitis in adults. Clin Infect Dis 2002;34:1440-8. 
Fig. 1. Median (interquartile range) duration of outpatient parenteral antibiotic therapy (OPAT) by year of presentation. 


\section{Table 1}

Baseline patient characteristics $(N=963)$

\begin{tabular}{ll} 
Characteristic & $n(\%)^{\mathrm{a}}$ \\
\hline Age (years) [median (IQR)] & $48(37-64)$ \\
Female sex & $396(41.1)$ \\
Penicillin allergy & $71(7.4)$ \\
Referred from community (GP or emergency department) & $604(62.7)$ \\
MRSA infection & $47(4.9)$ \\
Diabetes & $85(8.8)$ \\
Vascular disease & $30(3.1)$ \\
Immunocompromised $^{\mathrm{b}}$ & $49(5.1)$ \\
Infection type $^{\text {Cellulitis/erysipelas }}$ & \\
Bursitis, with or without cellulitis & $870(90.3)$ \\
Wound infection $_{\text {Infected ulcer }}$ & $29(3.0)$ \\
Other & $26(2.7)$ \\
Managed via a nurse-led PGD & $8(0.8)$ \\
\hline
\end{tabular}

IQR, interquartile range; GP, general practitioner; MRSA, meticillin-resistant

Staphylococcus aureus; PGD, patient group direction.

a Data are $n(\%)$ unless otherwise stated.

${ }^{\mathrm{b}}$ Malignancy, immunosuppressive therapy, human immunodeficiency virus (HIV) infection. 


\section{Table 2}

Outpatient parenteral antibiotic therapy (OPAT) outcomes, including reasons for readmission and nature of adverse events ${ }^{a}$

\begin{tabular}{llll}
\hline Outcome & Ceftriaxone $(n=$ & Teicoplanin $(n=$ & All $(N=$ \\
& $811)$ & $144)$ & $963)$ \\
\hline $\begin{array}{l}\text { Duration of OPAT in days } \\
\text { [median (IQR)] }\end{array}$ & $3(2-4)$ & $8(3-12)$ & $3(2-5)$ \\
Progression of infection & $21(2.6)$ & $6(4.2)$ & $27(2.8)$ \\
Re-admission & $43(5.3)$ & $15(10.4)$ & $58(6.0)$ \\
Planned admission & $13(1.6)$ & $3(2.1)$ & $16(1.7)$ \\
Logistics ${ }^{\text {b }}$ & $2(0.2)$ & $3(2.1)$ & $5(0.5)$ \\
Treatment failure & $14(1.7)$ & $5(3.5)$ & $19(2.0)$ \\
Non-device-related infection & $1(0.1)$ & $1(0.7)$ & $2(0.2)$ \\
Antibiotic reaction & $1(0.1)$ & 0 & $1(0.1)$ \\
Other medical complication & $12(1.5)$ & $3(2.1)$ & $15(1.6)$ \\
Significant adverse event & $45(5.5)$ & $21(14.6)$ & $68(7.1)$ \\
Rash & $10(1.2)$ & $13(9.0)$ & $25(2.6)$ \\
Severe allergy/anaphylaxis & $3(0.4)$ & 0 & $3(0.3)$ \\
Drug fever & 0 & $2(1.4)$ & $2(0.2)$ \\
Abnormalities of liver function & $8(1.0)$ & $3(2.1)$ & $11(1.1)$ \\
Renal dysfunction & $4(0.5)$ & $2(1.4)$ & $6(0.6)$ \\
Gastrointestinal & $15(1.8)^{c}$ & $1(0.7)$ & $16(1.7)$ \\
Other & $5(0.6)$ & 0 & $6(0.6)$ \\
OPAT failure & $85(10.5)$ & $37(25.7)$ & 124 \\
& & & $(12.9)$ \\
\hline & & &
\end{tabular}

IQR, interquartile range.

${ }^{\text {a }}$ Data are $n(\%)$ unless otherwise stated.

${ }^{\mathrm{b}}$ Antibiotic loading regimen, transportation problems, lack of carer.

${ }^{\mathrm{c}}$ Eleven episodes of diarrhoea, including one case of Clostridium difficile-associated disease. 


\section{Table 3}

Variables examined for an association with outpatient parenteral antibiotic therapy (OPAT) failure

\begin{tabular}{|c|c|c|c|c|}
\hline \multirow[t]{2}{*}{ Variable } & \multicolumn{2}{|l|}{$\begin{array}{l}\text { Univariate logistic } \\
\text { regression }\end{array}$} & \multicolumn{2}{|c|}{$\begin{array}{l}\text { Multiple logistic } \\
\text { regression }\end{array}$} \\
\hline & OR $(95 \% \mathrm{Cl})$ & $P$-value & OR $(95 \% \mathrm{Cl})$ & $P-$ \\
\hline $\begin{array}{l}\text { Age (per additional } 10 \\
\text { years) }\end{array}$ & $1.13(1.01-1.25)$ & 0.029 & & \\
\hline Female sex & $1.69(1.15-2.48)$ & 0.0070 & $\begin{array}{l}1.65(1.10- \\
2.47)\end{array}$ & 0.016 \\
\hline MRSA & $3.63(1.90-6.94)$ & $<0.0001$ & 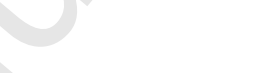 & \\
\hline Immunosuppression & $1.43(0.65-3.14)$ & 0.37 & & \\
\hline Diabetes & $2.26(1.30-3.92)$ & 0.0045 & $\begin{array}{l}2.02(1.12- \\
3.67)\end{array}$ & 0.020 \\
\hline Vascular disease & $3.24(1.44-7.29)$ & 0.005 & & \\
\hline Ischaemic heart disease & $1.94(0.71-5.31)$ & 0.20 & & \\
\hline Teicoplanin vs. ceftriaxone & $2.95(1.91-4.57)$ & $<0.0001$ & $\begin{array}{l}1.87(1.05- \\
3.33)\end{array}$ & 0.033 \\
\hline Referred from community & $\begin{array}{c}0.68(0.46- \\
0.996)\end{array}$ & 0.048 & & \\
\hline $\begin{array}{l}\text { Managed via PGD } \\
\text { Infection type }\end{array}$ & $0.57(0.39-0.83)$ & 0.0038 & & \\
\hline Bursitis vs. cellulitis & $\begin{array}{l}1.59(0.595- \\
4.27)\end{array}$ & 0.35 & & \\
\hline Infected ulcer vs. cellulitis & $\begin{array}{l}7.65(1.88- \\
31.07)\end{array}$ & 0.0044 & & \\
\hline $\begin{array}{l}\text { Wound infection vs. } \\
\text { cellulitis }\end{array}$ & $3.60(1.52-8.56)$ & 0.0037 & & \\
\hline Other infection vs. cellulitis & $1.66(0.62-4.47)$ & & & \\
\hline
\end{tabular}


OR, odds ratio; $\mathrm{Cl}$, confidence interval; MRSA, meticillin-resistant Staphylococcus aureus; PGD, patient group direction. 


\section{Table 4}

Variables examined for an association with duration of outpatient parenteral antibiotic therapy (OPAT) therapy

\begin{tabular}{|c|c|c|c|c|}
\hline \multirow[t]{3}{*}{ Variable } & \multicolumn{2}{|l|}{$\begin{array}{l}\text { Univariate linear } \\
\text { regression }\end{array}$} & \multicolumn{2}{|c|}{ Multiple linear regression } \\
\hline & \multirow{2}{*}{$\begin{array}{l}\text { Estimate (95\% } \\
\mathrm{Cl})^{\mathrm{a}}\end{array}$} & \multirow[t]{2}{*}{$P$-value } & \multicolumn{2}{|c|}{ Estimate $(95 \% \quad P$-value } \\
\hline & & & $\mathrm{Cl})^{\mathrm{a}}$ & \\
\hline $\begin{array}{l}\text { Age (per additional } 10 \\
\text { years) }\end{array}$ & $1.07(1.05-1.10)$ & $<0.0001$ & $1.03(1.01-1.05)$ & 0.0097 \\
\hline Female sex & $1.05(0.96-1.15)$ & 0.30 & & \\
\hline MRSA & $3.06(2.53-3.72)$ & $<0.0001$ & $1.47(1.17-1.84)$ & 0.0010 \\
\hline Immunosuppression & $1.50(1.22-1.83)$ & 0.0001 & & \\
\hline Diabetes & $1.24(1.06-1.45)$ & 0.007 & & \\
\hline Vascular disease & $1.93(1.50-2.48)$ & $<0.0001$ & $1.29(1.01-1.64)$ & 0.041 \\
\hline Ischaemic heart disease & $1.52(1.14-2.03)$ & 0.0040 & & \\
\hline Teicoplanin vs. ceftriaxone & $2.09(1.86-2.34)$ & $<0.0001$ & $1.32(1.16-1.50)$ & $<0.0001$ \\
\hline Referred from community & $0.77(0.70-0.84)$ & $<0.0001$ & $0.91(0.84-0.99)$ & 0.021 \\
\hline Managed via PGD & $0.58(0.53-0.62)$ & $<0.0001$ & $0.71(0.65-0.77)$ & $<0.0001$ \\
\hline \multicolumn{5}{|l|}{ Infection type } \\
\hline Bursitis vs. cellulitis & $1.89(1.49-2.40)$ & $<0.0001$ & $1.81(1.45-2.25)$ & $<0.0001$ \\
\hline Infected ulcer vs. cellulitis & $3.17(2.02-4.98)$ & $<0.0001$ & & \\
\hline $\begin{array}{l}\text { Wound infection vs. } \\
\text { cellulitis }\end{array}$ & $3.73(2.88-4.82)$ & $<0.0001$ & $1.74(1.31-2.30)$ & 0.0001 \\
\hline $\begin{array}{l}\text { Other infection vs. } \\
\text { cellulitis }\end{array}$ & $1.38(1.08-1.76)$ & 0.0091 & $1.25(1.00-1.56)$ & 0.0049 \\
\hline
\end{tabular}

$\mathrm{Cl}$, confidence interval MRSA, meticillin-resistant Staphylococcus aureus; PGD, patient group direction.

${ }^{a}$ Estimates: percentage change in number of days in OPAT, e.g. an estimate of 1.10 means that, on average, a variable is associated with a $10 \%$ increase in the number of days of treatment. 


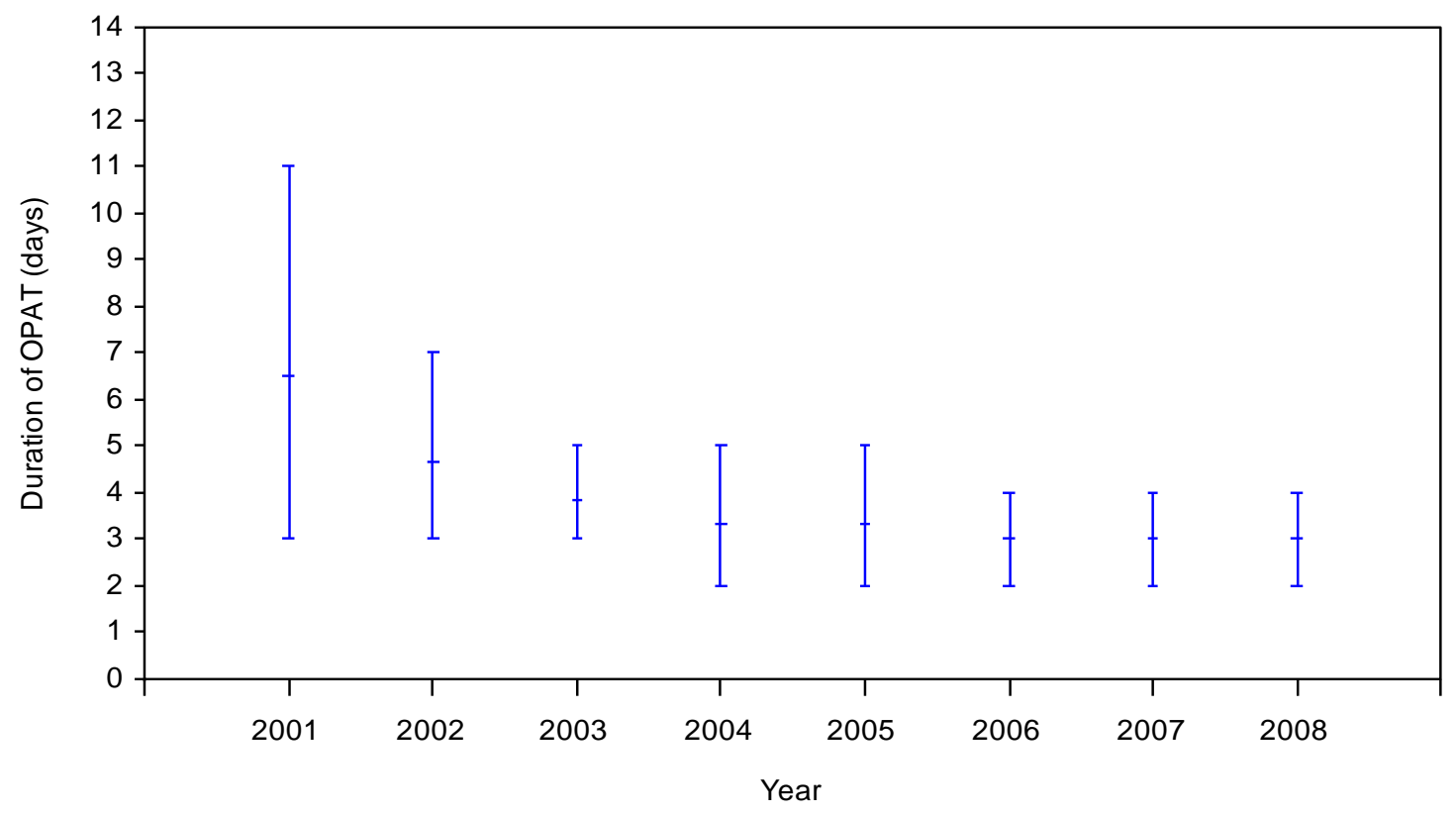

Data shown are median, lower quartile and upper quartile 